\title{
Airfoil cascades with bistable separation control
}

\author{
V. Tesă̌ \\ Institute of Thermomechanics v.v.i., \\ Academy of Sciences of the Czech Republic, Czech Republic
}

\begin{abstract}
To accommodate varying flow rate and shaft speed conditions, the airfoils arranged in cascades in hydrodynamic machines (turbines, pumps) should be variable. Mechanical articulation is expensive and complex, reducing reliability. The author investigated separation control by jets issuing from fixed stator blades shaped so that the flow past them is switched by short control-jet pulses between two alternative regimes differing in the resultant exit flow angle. Although applicable widely, in the tests the stator cascades were mostly used in simple rotor-less devices operating as a vortex-flow restrictor. Experiments were supplemented by extensive numerical flowfield solutions.
\end{abstract}

Keywords: separation control, airfoil cascade, Coanda effect.

\section{Introduction: the role of airfoil cascades}

Cascades, the periodic systems of airfoils, e.g. Scholz [1], are essential components of hydrodynamic machines, such as pumps or turbines. In these two machine types, energy conversion in one direction takes place, e.g. mechanical output is produced at the expense of decreasing hydraulic energy. The conversion actually usually involves two cascades, a fixed stator cascade and the other on the rotor connected with the rotating shaft. The energy transfer between the fluid flow and the mechanical work takes place in the rotor cascade. The stator (sometimes missing in machines that have to be cheap) either directs the flow into the turbine rotor or, in a pump or blower, converts the kinetic energy gained by the fluid in the rotor into the pressure rise. More complex conversions take place in machines with a pair of rotor cascades, each on its shaft (usually each also having its corresponding stator cascade). One of them has the role of a 
turbine and the other operates as a pump. With mechanical power transfer by a shaft connecting the two rotors, it is the case of a turbo-charger. On the other hand, in couplings and torque converters it is the fluid flow that transfers the power from the driving shaft to the driven one. There are also less common machines, such as retarders; in principle hydrodynamic couplings with fixed or absent output shaft, used to dissipate the mechanical input power. The flow restrictors have no rotor. They dissipate the input hydraulic power by vortex flow in a chamber located downstream from the stator.

Operating conditions of turbomachines are determined mainly by fluid flow rate and shaft rotational speed. In many applications they vary, sometimes in a wide range. Geometry of airfoils is designed for a particular working condition at which their efficiency attains a maximum. Deviations from this regime inevitably change the attack angle at which the fluid enters the cascade. This results in efficiency deterioration, especially if the attack angle is large enough for the airfoils to stall. Usually, the airfoil shapes are designed with some compromising so as to accept attack angle changes within a certain range, though the extent of this range is inevitably limited.

Acceptable efficiency, even with large variations of the flow rate, may be maintained- at least to a degree - by articulated vanes or blades. The standard solution (Fig. 1) is to vary the vane attack angle by mechanical actuators. Each vane is held in its bearing and all are connected to some actuation mechanism. This makes the machine more expensive - and yet the conditions cannot be ensured to remain ideal at all vane angular positions (this would require varying the vane shape and not just the attack angle). Nevertheless, efficiency

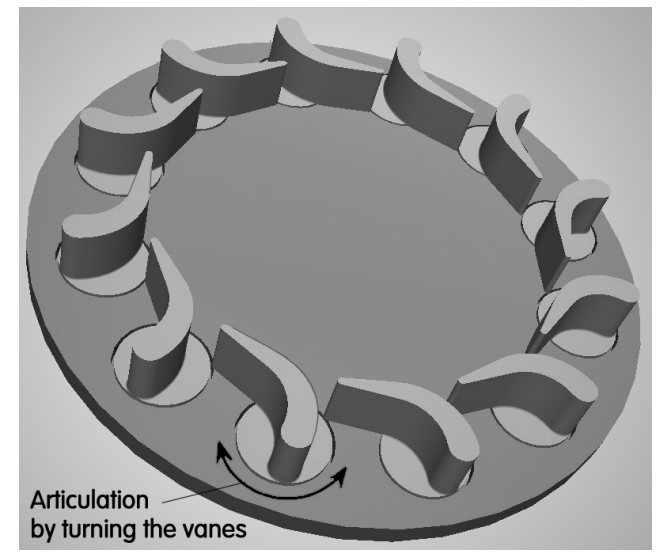

Figure 1: An example of a stator cascade with the classical mechanical articulation by turning the vanes. The necessary vane bearings and the actuation levels make this solution expensive and acceptable only for very large or very important turbo-machines. In the discussed solution, the vanes are fixed and their aerodynamic properties are varied by auxiliary control fluid flows. 
improvement over non-articulated vanes is substantial. Apart from the compensation for flow rate or shaft speed variations, the actuator mechanism may serve as an input controlling the machine operation. In general, the mechanical articulation is justified only in machines handling large power levels. This is an unpleasant fact especially for very small turbomachines, belonging to microfluidics, e.g. Tesař [7]. Due to the small Reynolds number, efficiency of a microturbine is the critical factor and keeping it reasonably high by actuation of cascades would be particularly welcome.

Since there is a certain range of acceptable attack angles, a design capable of operating in two regimes is often acceptable. This may simplify the actuation somewhat. In the machines for power transfer between two shafts there is a solution using a gearbox connected to one of the shafts. This is not uncommon in car automatic transmissions, where it is acceptable (in spite of the gearbox cost) because of the long tradition of gearboxes in automobiles (and availability of the machine tools for manufacturing them). Experience shows that only two gear pairs may suffice despite the wide range of the car operating conditions.

\section{Fluidic control of cascade flow}

Characteristic feature of fluid flow control in aerodynamics is replacing the early solutions - with typical mechanical linkages and movable flaps or spoilers protruding into the flow - by fluid jets. This inspired author's investigations into the possibilities of similar development in the turbomachine cascades. The example in Fig. 2 is a historical early attempt (Tesař [2]) to replace mechanically articulated stator blades of a torque converter. In response to the control flows from the slit nozzles in the hollow blades, two flow regimes are possible. They
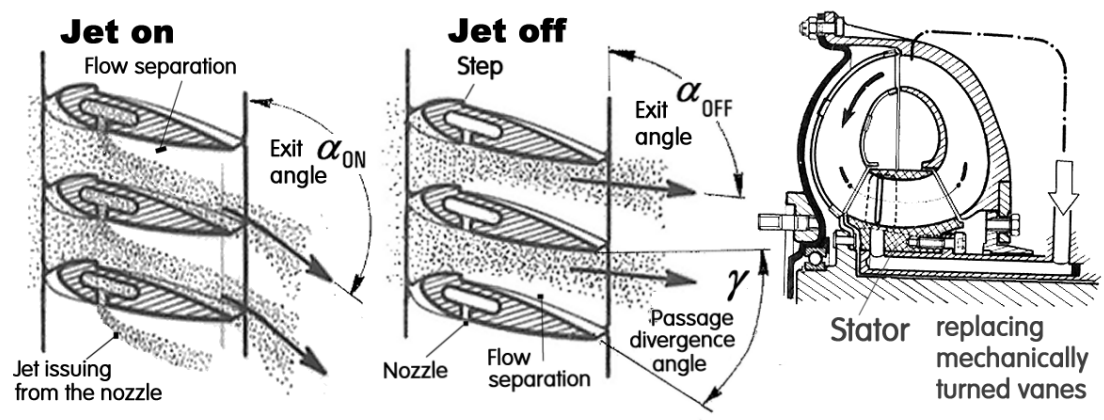

Figure 2: Cascade of airfoils with controlled separation in the passage between the blades. Divergence angle $\gamma$ is too large for attachment to both walls. Without the control jets (centre), the flow separates (assisted by the step) from the dorsal wall. Jets from the slit nozzles (left) create the other regime, with the flow separating from the ventral side and leaving the cascade at a much larger angle $\alpha_{0 N}$. 
differ in the angle $\alpha$ at which the flow leaves the cascade. The flow thus enters the rotating (pump) cascade immediately downstream at different angles of attack. If the rotor speed reaches a level at which, in the absence of the control flow, the attack angle is too large and the flow would separate from its blades, the separation is suppressed by switching the stator cascade to the other regime, with smaller attack angles.

The more recent designs - Fig. 3 presents an example of an application in the stator of a small radial centripetal turbine - retained the basic features of their forerunner from Fig. 2: the channel between the vanes causes the main flow to attach to one of the two walls further downstream. There are two regimes $A$ and B. The inlets for the control flow are in the vanes, which tend to be unusually thick (a typical feature of airfoils with separation control). The fact that only two regimes are possible, and also that in both there is some separation, admittedly relegates this idea to less important machinery, especially of small size. It is an advantage that the fixed blades are much easier and cheaper to manufacture than their mechanically adjusted equivalents. Of course, availability of the control fluid flow, another limiting factor, is seldom a problem.

Later, essentially the same idea was applied to a valve presented Fig. 4. It was to be used in a filter for removal of particulate emissions from Diesel engine exhaust and operated at rather high temperatures. The filter clogs rather fast and has to be re-generated by burning the deposited carbon particles. During the regeneration the gas flow rate has to be decreased. This is achieved by temporary by-passing of the main gas flow through this turn-down valve. Designed according to Figs. 5 and 6 , the valve contains no moving components and is therefore eminently suitable for the demanding conditions of operation at 350 $600{ }^{\circ} \mathrm{C}$.

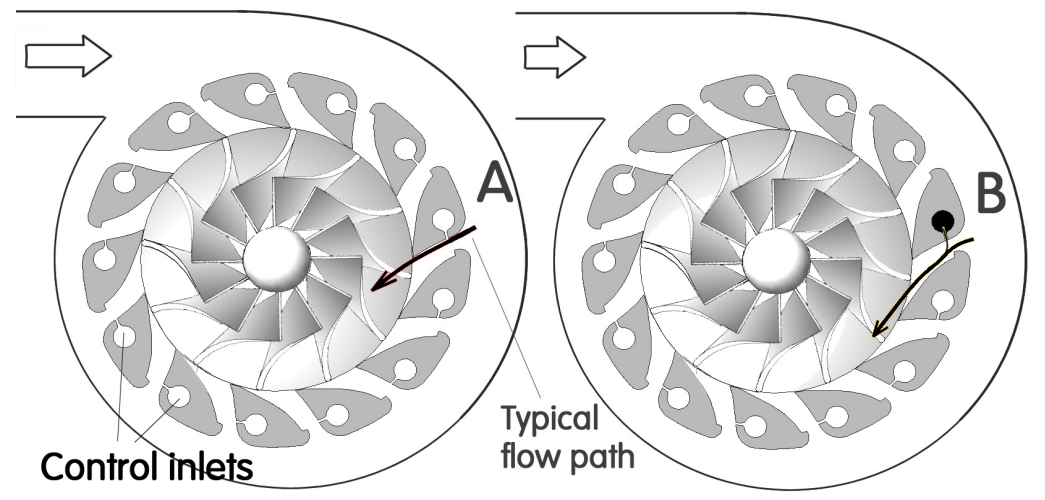

Figure 3: No-moving-part stator proposed for a small radial turbine. There are only two regimes (control flow ON or OFF). In both, the flow separates from one of the vane walls. Efficiency is better than with non-articulated vanes. 
In this case, the cascade is bistable (while those in Figs. 2 and 3 were monostable). Again, the airfoils are rather thick - a feature common in airfoils with separation control by jets. They form an axial-flow stator that directs the flow passing between them either radially or tangentially into the vortex chamber. The divergence angle between the walls $\mathbf{m}$ and $\mathbf{n}$ (Fig. 6)

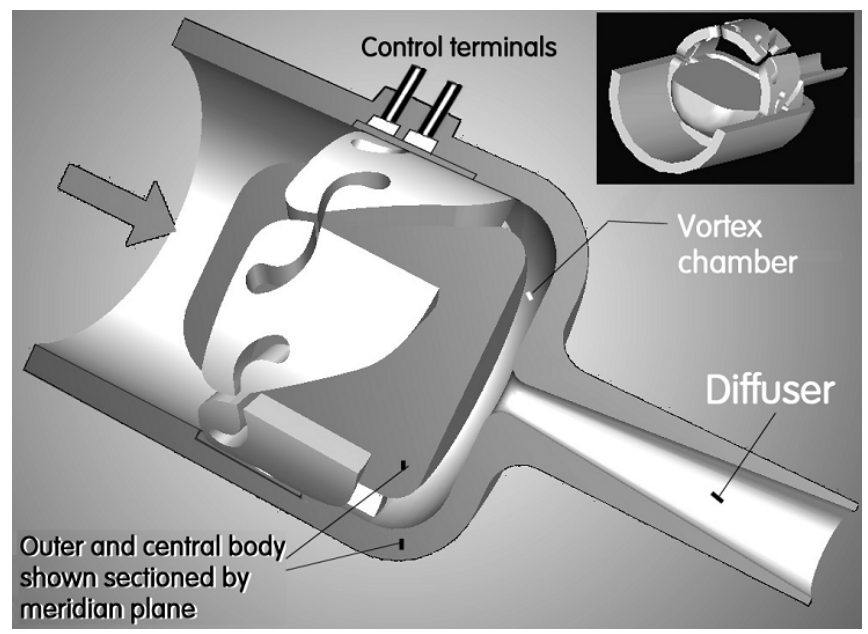

Figure 4: Jet-switched, axial flow stator cascade used to control the entry into vortex chamber in a no-moving-part turn-down valve, originally designed to reduce the Diesel engine exhaust gas flow in the regeneration phase of particulate emissions filter.

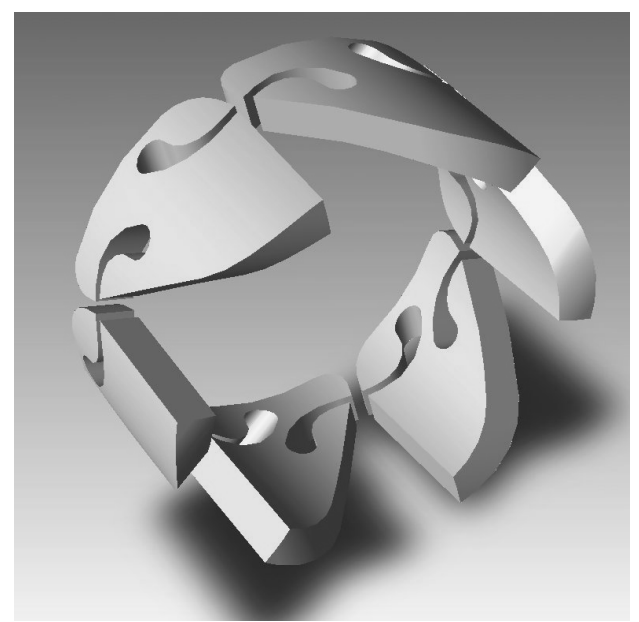

Figure 5: The stator cascade of six very thick, very short span airfoils from the previous Fig. 4. There is a control nozzle on both sides of the gap between the airfoils. 


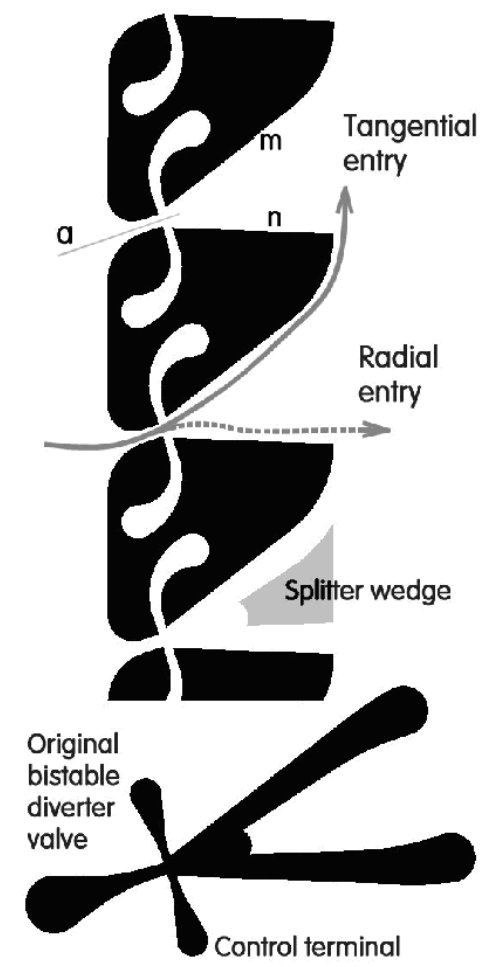

Figure 6: The cascade compared with (at the bottom) author's fluidic bistable valve VT30 described in by Tesař $[4,5]$. The shape of the airfoils was actually designed starting from the geometry of the gaps between them.

is here as large as $\gamma=40^{\circ}$, symmetric with respect to the entrance axis a. The flow attaches either to the wall $\mathbf{m}$ or to $\mathbf{n}$. If attached to $\mathbf{m}$ the flow enters the vortex chamber tangentially and its rotation velocity increases as the fluid proceeds towards the exit diffuser throat. The centrifugal effect then nearly stops the flow. If the flow is switched to attach to $\mathbf{n}$, it passes radially through the vortex chamber. Without rotation, the pressure drop across the valve in this "open" state is small.

The airfoil design started from the geometry of the gap between them, using earlier author's experience [3-5] with the bistable fluidic diverter valves, as shown in Figs. 7, 8, and 9. Because the attachment to any of the two mutually opposing walls is stable, considerable saving is achieved of the control flow. For the switching, only very short control flow pulses suffice.

The problem with the valve of Fig. 4 was the complexity of the spatial geometry of the airfoils, Fig. 5, difficult to manufacture on standard machine tools when building the one-off laboratory model. This has led to the attention 


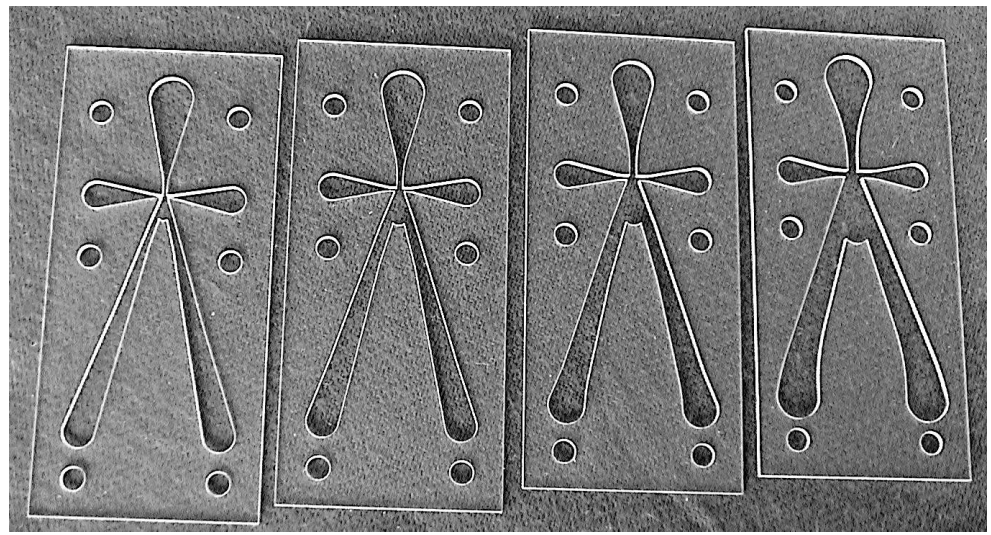

Figure 7: Perspex plates with family (note the different size of outlet diffusers) of laser-cut fluidic bistable unvented valves.

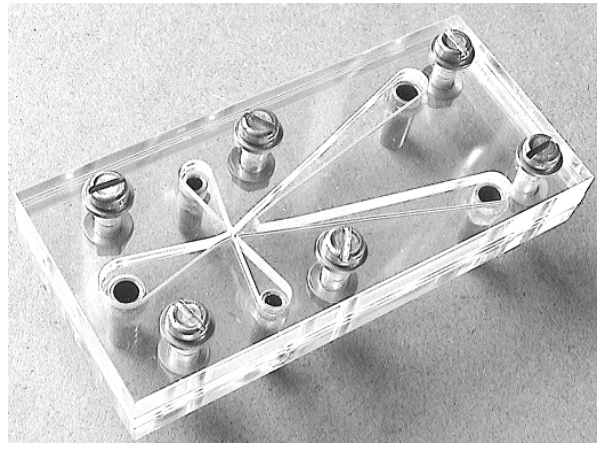

Figure 8: Laboratory model of Figure 9: author's fluidic diverter valve [3].

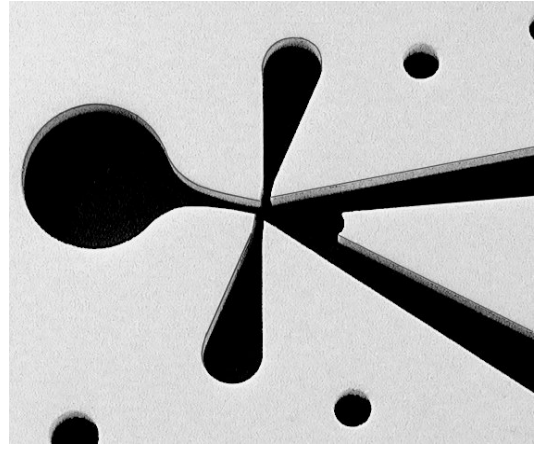

A detail of the key parts of the valve, the cavity for Coandaeffect attachments and interaction of the flows - in this case cut in Teflon [10].

later changed to the version with planar radial-flow cascade shown in Fig. 10, much easier to make - Fig. 11. It is again a rotor-less vortex valve. Because of most of its operating time spent in the CLOSED, tangential entry regime (the turning down is not complete), there is again the monostable behaviour. In contrast to the symmetric attachment walls in Fig. 6, here (Fig. 11) the preferential attachment wall $\mathbf{m}$ is aligned with the entrance axis a - while the other wall $\mathbf{n}$ was not only inclined at a large deviation angle but also separated by the large setback step. The advantage gained is the simplified control, needing 


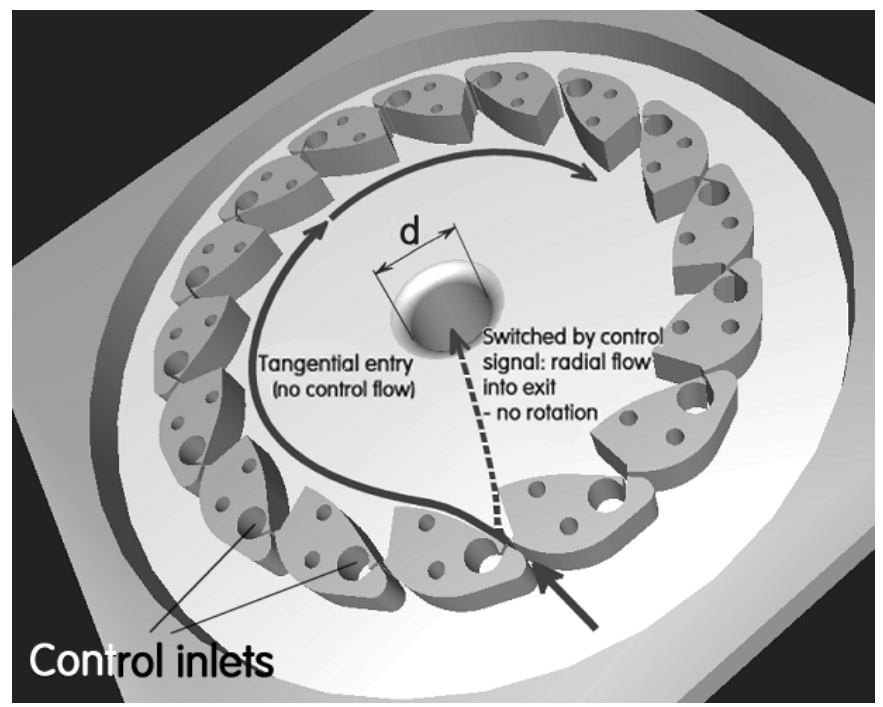

Figure 10: A cascade of the author's radial-flow airfoils in a monostable restrictor. The flow rotates so that the centrifugal acceleration turns it down - or is switched by control flows into the OPEN lowresistance state with radial entry into the vortex chamber.

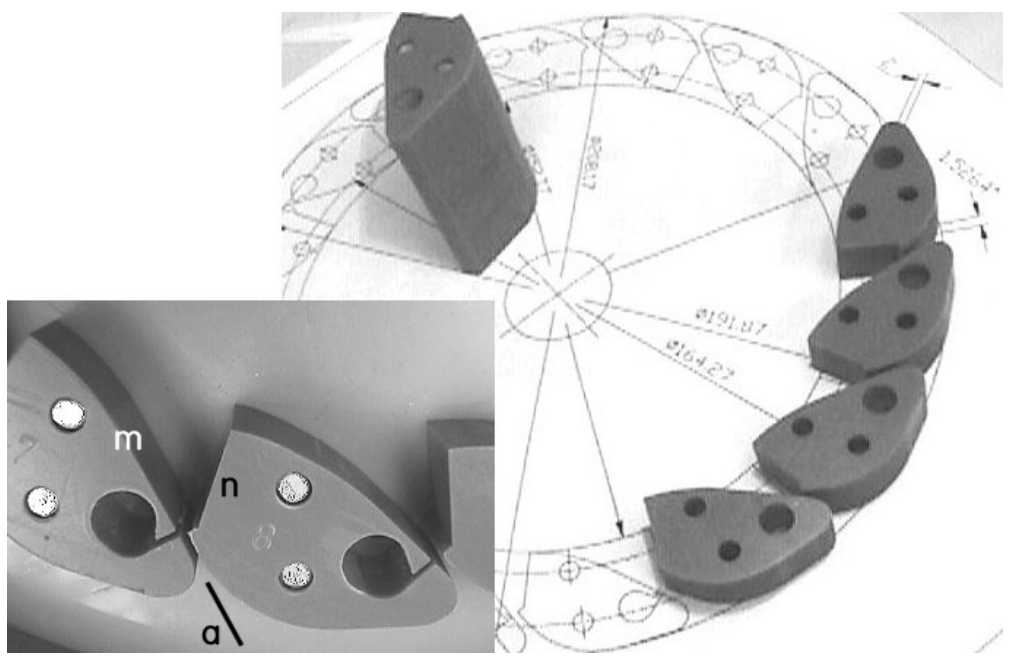

Figure 11: Left bottom corner: detail photograph of neighbouring airfoils (their punched workshop numbering may be noted), each held by a pair of steel pins. On the right, positioned on the workshop drawing, are airfoils during their manufacturing. Control nozzle slits were not yet made at this stage. The outer shapes were milled simultaneously in the stack of all 16 blades. 
just a single control inlet. Of course, the attachment to $\mathbf{n}$ requires permanent control fluid flow to keep the valve OPEN. This was acceptable in the application to the Diesel engine filter where short re-generations (with the vortex valve open) take place between long-duration periods of sooth removal, during which no compressed air flow for the valve control is needed.

The monostability is obtained by giving to the wall $\mathbf{n}$ a large $41 \mathrm{deg}$ deviation angle and also by separating it by the $1.8 \mathrm{~mm}$ separation step. The airfoils were cut from polyvinyl chloride plate $15 \mathrm{~mm}$ thick (nominally). The admittedly rather inefficient shape of the control nozzles was dictated by the available area inside the airfoils for placing the $9.8 \mathrm{~mm}$ dia circular control inlet chambers $\mathrm{X}$ and also by the method of cutting the nozzle slit. In another tested alternative, Fig. 12, controlling the main flow and not the by-pass, the role of the control was reversed: the flow into $X$ is needed to keep the valve CLOSED during the regeneration. The performance in this version is somewhat better due to the flow from the control nozzle in the CLOSED regime interacting with the main flow in the OPEN state. There are two dominant cross sections in these restrictors: (a) the entrances into the inter-blade passages, of cross section bh (Fig.5), and (b) the vortex chamber exit. An efficient centrifugal action in the CLOSED regime requires the exit diameter to be as small as possible. On the other hand, the OPEN state requires a large exit area - or more exactly low dissipance [7, 14] of the exit, which may be achieved even with a small diameter by placing downstream from the exit a diffuser. An important design problem was the proper mutual relation between the two dominant cross sections. Early experiments suggested the optimum is reached with both areas equal. This remains a useful rough "rule

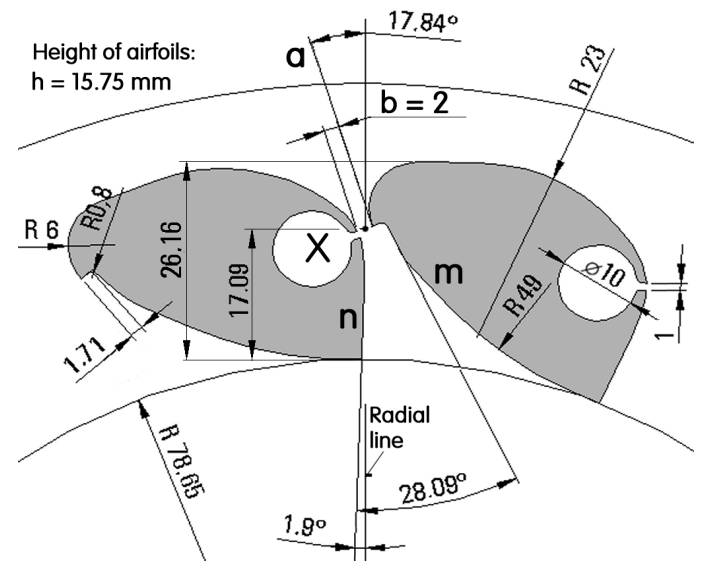

Figure 12: Geometry of the very thick airfoils used in another monostable variant of the vortex restrictor valve. In this case (note the position of the control $\mathrm{X}$ relative to the attachment walls $\mathrm{m}$ and n) the control flows switch the valve into the CLOSED, highresistance regime. 
of thumb", but a detailed discussion of the more exact solution of this matching problem is now available, using the approach discussed in Tesar [5]. Investigations performed with the monostable vortex restrictor having the cascade of the geometry shown in Fig. 12 and the exits as shown in Fig. 13 have shown the optimum conditions attained (Fig. 16) with the area of the exit diffuser throat somewhat smaller - only $76 \%$ of the total area 16 bh of the entrances. As with the vortex-type non-return valves [6],

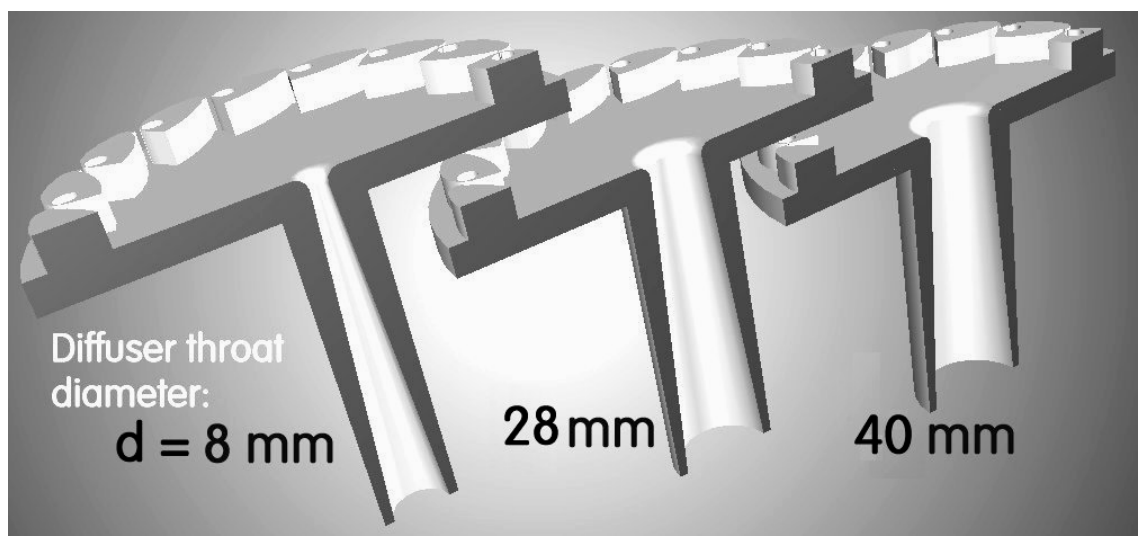

Figure 13: Investigations made to find the matching the cascade properties with those of the vortex-chamber exit. Various alternative exit diffusers were of identical $170 \mathrm{~mm}$ length.

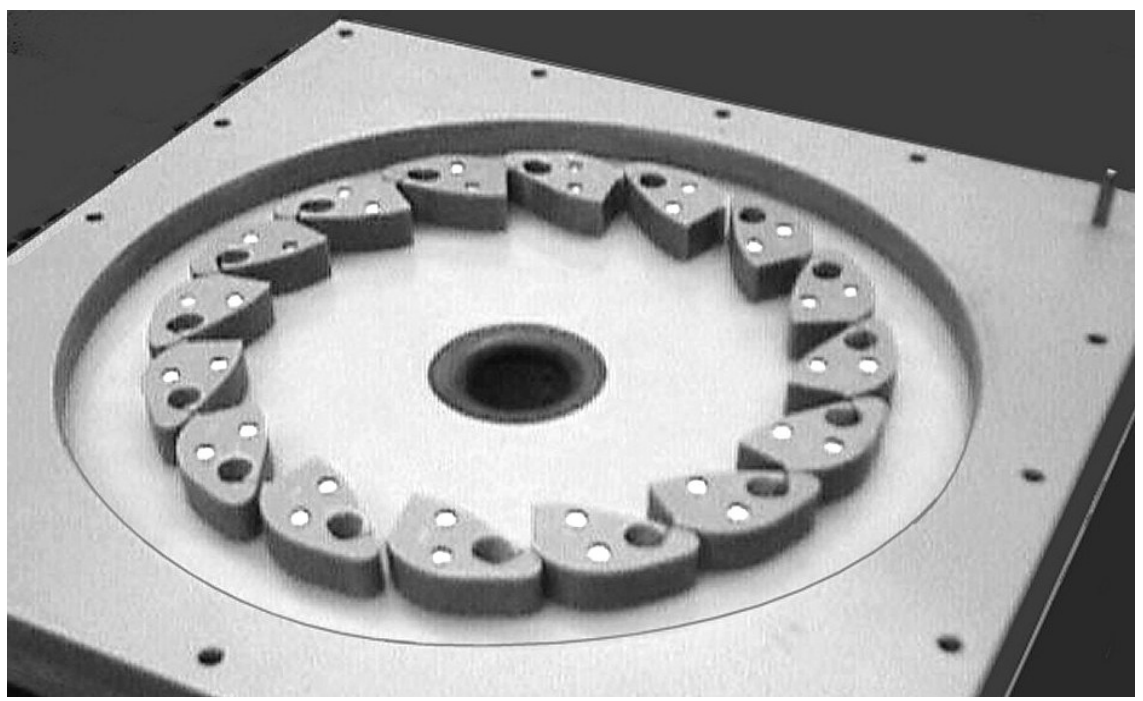

Figure 14: Photograph of the assembled monostable turn-down valve model with 16 airfoils and a large-diameter exit diffuser. 
the turning-down action depends on the flow dynamics so that complete closure is not possible. Nevertheless, the demonstrated decrease shown in Fig. 16 to the value representing mere $3.6 \%$ of the magnitude in the OPEN state at the same pressure represents a good performance value, no doubt sufficient for most potential applications.

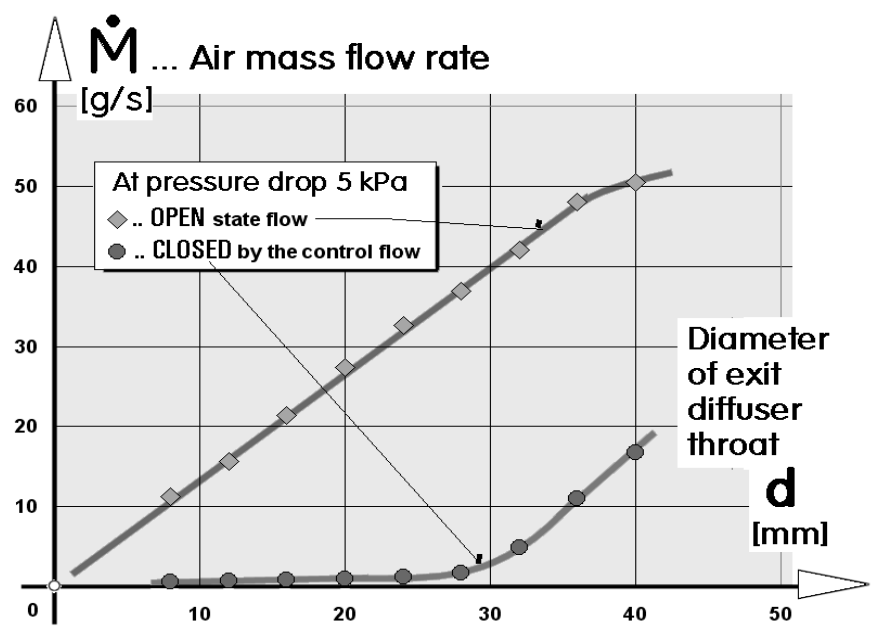

Figure 15: The two dependences on the exit diameter (the OPEN and CLOSED regimes) of the air flow rate through the restrictor with airfoils according to Fig. 12 at the same overall pressure drop $5 \mathrm{kPa}$.

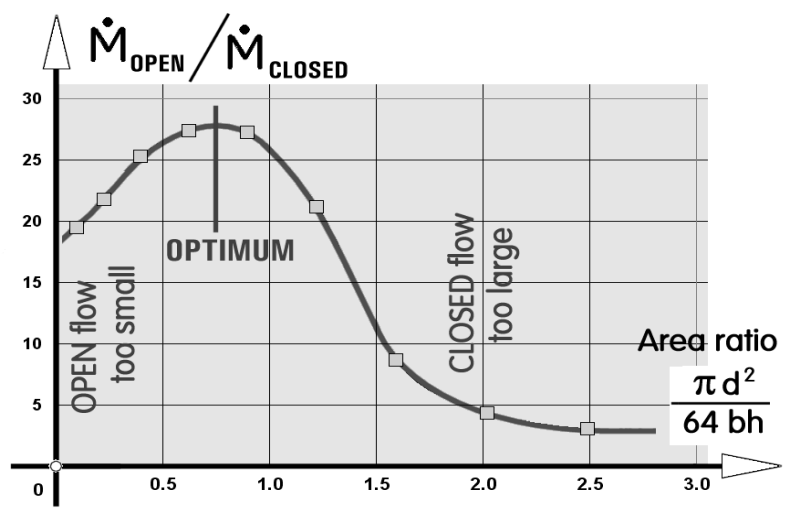

Figure 16: Ratio of flow rates in the two regimes at the same pressure drop, plotted as a function of the ratio of the two dominant cross section areas: the exit area and the area of the smallest cross sections in the channels between the airfoils. 


\section{Conclusions}

Flow control is currently receiving most attention in the field of fluid dynamics. Apart from the control logic and sensor systems, considerable work is needed on the actuator side - devices enacting large-scale changes in the flowfield in response to small control signals, preferably exhibiting high reliability, robustness and low cost. Early developments based on mechanical linkages and movable bodies protruding into the flow tend to be replaced action of fluid jets. Most of this activity is currently in external aerodynamics - mainly for improving performance of flight vehicles. Present paper turns the attention to applying analogous ideas to internal aerodynamics - namely to airfoil cascades, the essential parts of energy converting hydrodynamic machines.

\section{Acknowledgements}

Gratefully acknowledged is support by grant IAA200760705 and by the research plan AV0Z20760514 received from the Grant Agency of the Academy of Sciences of the Czech Republic, and by the grant 101/07/1499 from GACR Czech Science Foundation.

\section{References}

[1] Scholz N., Aerodynamics of Cascades - in German, Braun, Karlsruhe, 1965

[2] Tesař V., Machine converting energy between a mechanical motion and a fluid flow - in Czech language, Czechoslovak Patent Nr. 230175, filed 10th April 1981

[3] Tesař V., Hung C.-H., Zimmerman W.: No-Moving-Part HybridSynthetic Jet Actuator, Sensors and Actuators A, Vol. 125, p. 159, 2006

[4] Tesař V., Mechanism of Pressure Recovery in Jet-Type Actuators, Sensors and Actuators A-Physical, Vol. 152, p.182, 2009

[5] Tesař V., Fluidic control of reactor flow - Pressure drop matching, Chemical Engineering Research and Design, Vol. 87, p. 817, 2009

[6] Thoma D., Non-return valve - in German, VDI-Zeitschrift, Band 74, p. 1098, 1930.

[7] Tesař V., "Pressure-Driven Microfluidics", Artech House Publishers, Norwood, MA, USA, 2007

[8] Tesař V., "Bistable turn-down power amplifier - Matching the two dominant cross sections", Proc. of the 9-th 'Jablonna' International Fluidics Conference, Paper A-5, Jablonna, Poland, Sept. 1982

[9] Tesař V.: "Arrangement for controlled transfer of rotational motion" - in Czech language, Czechoslovak Patent Nr. 225603, filed 10th April 1981

[10] Tesař V.: “Enhancing Impinging-Jet Heat or Mass Transfer by Fluidically Generated Flow Pulsation", Chemical Engineering Research and Design, Vo. 87, p. 181, 2009I 
[11] Tesař V.: "Fluidic Valves for Variable-Configuration Gas Treatment", Chemical Engineering Research and Design, Vol. 83 (A9), p. 1111, 2005

[12] Tesař V.: "Fluidic Valve for Reactor Regeneration Flow Switching", Chemical Engineering Research and Design, pp.1 -11, Part A, Vol, 82 (A3), 2004

[13] Tesař V.: "Großmaßstäbliche fluidische Ventile für die Durchflußsteuerung" (Large-scale fluidic valves for flow control - in German), Messen-steuern-regeln Bd. 26, Nr. 41983

[14] Tesař V.: "Characterisation of three-terminal fluidic elements and solution of bifurcated-flow circuits using the concept of equivalent dissipance", Journal of Fluid Control / Fluidics Quarterly, Vol. 13, Nr. 2, June 1981

[15] Tesař V.: "Valvole fluidiche senza parti mobile" (Fluidic valves without moving parts - in Italian), Oleodinamica - pneumatica, pp. 216-223, No 3 - Anno 39, marzo 1998 